\section{KOMPASS}

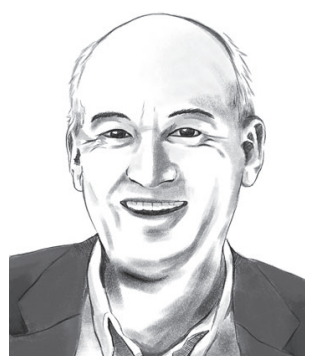

Harald Walach

Poznan/Berlin,

Polen/Deutschland

Political Correctness (PC) kommt mir manchmal vor wie die neue Tyrannei. Bevor ich vom Leder ziehe ein paar anerkennende Worte. PC ist die moderne Form des ungeschriebenen Gesetzes. Alle Gemeinschaften, Familien, Arbeitsgruppen, Kliniken, Universitäten, Kulturbetriebe und Nationen kennen solche ungeschriebenen Gesetze. Diese sind wahrscheinlich noch wichtiger als die geschriebenen. Denn sie regeln das Zusammenleben im Detail. Deswegen muten wir andern nicht zu, unsere Fäkalien wegzuräumen und greifen nicht einfach dem Nachbarn am Nebentisch in den Teller, obwohl uns dessen Essen vielleicht besser gefällt als das Eigene. Wenn sich einer nicht an diese ungeschriebenen Regeln hält, dann geht es inm meistens schlecht. Er wird ausgestoßen oder marginalisiert. Bei Eingeborenenstämmen waren diese Regeln das Tabu, und Tabubrecher wurden verurteilt. Bei uns nennen sich diese Regeln heute Sitte, Brauchtum, und, ja, mittlerweile eben auch PC.

Zusätzlich zu den Benimmregeln, die unser Zusammenleben einfach und reibungslos machen, gibt es heute, vielleicht sogar zunehmend mehr, Denkregeln, Glaubenssätze und geistige Tabus, denen wir uns unhinterfragt und sehr oft auch unbemerkt unterwerfen. Das ist der Bereich von PC. Da gibt es Denkvorschriften, die, wenn sie durchbrochen werden,

\title{
Political Incorrectness
}

rasch zum persönlichen Problem für den postmodernen Tabubrecher werden können. Ein gutes Beispiel ist Peter C. Gøtzsche, der ehemalige Leiter des Nordic Cochrane Centers in Kopenhagen. Er war Mitbegründer dieser allseits bekannten und geschätzten Institution, hat mehr als 100 Aufsätze in 4 großen medizinischen Journalen publiziert. Und er hat dreimal gegen $\mathrm{PC}$ verstoßen.

\section{Jedes System braucht Korrektive, um \\ bestehende Denkvorschriften zu hinterfragen.}

Zuerst, indem er der pharmazeutischen Industrie vorgeworfen hat, sich wie die Mafia zu benehmen [1]. Dann, indem er der Psychiatrie vorgeworfen hat, sie würde sich von der Industrie korrumpieren lassen und wissentlich mit gefährlichen und unbrauchbaren Substanzen arbeiten [2]. Und schließlich, indem er den Cochrane Review über die Effektivität der HPV-Impfung zur Prävention des Zervixkarzinoms zerrissen hat, weil dieser nur die Hälfte aller Daten berücksichtigt hätte und die Autoren gegen Cochrane Regeln zur Vermeidung von Interessenskonflikten verstoßen hätten [3, 4]. Gøtzsche wurde auf Betreiben des CEO von Cochrane aus seiner Stelle als Internist am Reichskrankenhaus in Kopenhagen entfernt und geht dagegen mit rechtlichen Mitteln vor [4]. Konsequen- terweise hat Gøtzsche nun ein eigenes «lnstitute for Scientific Freedom» gegründet. Das Beispiel zeigt, dass wir immer noch die gleichen Mechanismen anwenden wie altertümliche Volksstämme: Wir verstoßen den Tabubrecher, anstatt zu überlegen, wo unsere Denkvorschriften und Benimmregeln vielleicht in der Tat wenig dienlich sind. Ja, Tabubrecher und PC-Renegaten sind unbequem.

Doch jedes System braucht Korrektive, um bestehende Denkvorschriften zu hinterfragen. Daher sollten wir die modernen Tabubrecher, die bewusst oder unbewusst gegen PC verstoßen, willkommen heißen. Auch wenn es sehr unbequem ist, auch wenn sie nicht immer in allem recht haben, auch wenn sie unsere Routine stören. Denn möglicherweise sehen sie ja eine wichtige Wahrheit, die alle anderen übersehen. Solche PCTorpedos gibt es in jeder Organisation, in jede Arbeitsgruppe. Zum Glück! Heißen wir sie willkommen als ein Geschenk des Universums, um uns vor Verbohrtheit, intellektueller Öde und zwischenmenschlichem Tod zu schützen.

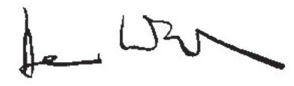

Prof. Dr. Dr. phil. Harald Walach

4 Gøtzsche PC. Death of a Whistleblower and Cochrane's Moral Collapse. Copenhagen: People's Press; 2019.
1 Gøtzsche PC. Deadly Medicines and Organised Crime: How Big Pharma Has Corrupted Health Care. London: Radcliff; 2013.

$\checkmark 2$ Gøtzsche PC. Deadly Psychiatry and Organised Denial. Copenhagen: People’s Press; 2015.
3 Jørgensen L, Gøtzsche PC, Jefferson T. The Cochrane HPV vaccine review was incomplete and ignored important evidence of bias. BMJ Evidence-Based Medicine 2018; 23(5): 165-8.

\section{KARGER}

Fax +497614520714 information@karger.com www.karger.com (c) 2019 S. Karger GmbH, Freiburg

Accessible online at: www.karger.com/kkd
Prof. Dr. Dr. phil. Harald Walach

CHS Institut

Schönwalder Straße 17

13347 Berlin, Deutschland 